\title{
Understanding c-di-GMP Signaling Specificity in Pseudomonas aeruginosa Pel- dependent Biofilms
}

\author{
J Van Loon ${ }^{1}$, P Howell ${ }^{1}, \mathrm{G}$ Whitfield ${ }^{2}$ \\ ${ }^{1}$ Program in Molecular Medicine, The Hospital for Sick Children, ${ }^{2}$ Department of Biochemistry, \\ University of Toronto, Toronto, Ontario \\ jaime.vanloon@mail.utoronto.ca
}

Background: Biofilms are communities of microbial cells surrounded by an extracellular matrix (ECM) composed primarily of exopolysaccharides (EPS), protein and extracellular DNA (1). The ECM is an important virulence factor that enables the embedded microbes to resist the immune system and antibiotic therapies (2). The opportunistic Gram-negative pathogen Pseudomonas aeruginosa predominantly exists as a biofilm and is commonly found in chronic infections, such as in the lungs of Cystic Fibrosis (CF) patients (3). The Pel polysaccharide is a major contributor to the ECM of clinical P. aeruginosa CF strains. The production of Pel requires the proteins encoded by the pelABCDEFG operon (Figure 1A) $(4,5)$ and its synthesis is positively regulated post-translationally by the binding of cyclic-3',5'-dimeric guanosine monophosphate (c-di-GMP) to PelD (6). Levels of c-di-GMP in the cell are modulated by the opposing activities of diguanylate cyclases and phosphodiesterases (PDEs) (7). As there are $>40$ c-di-GMP metabolic enzymes present in P. aeruginosa, the mechanisms by which signalling specificity is achieved remain largely unknown. Hypothesis: c-di-GMP metabolic enzymes achieve their specificity by interacting directly with c-di-GMP receptors causing conformational changes that effect function. Results: Using coimmunoprecipitation, mass spectrometry and bacterial two-hybrid approaches we have found that PelD directly interacts with the PDE BifA, which is known to specifically down-regulate Pel biosynthesis (8). Bioinformatics analyses predict that BifA has a periplasmic sensory HmsP domain that is connected to a dual degenerate diguanylate cyclase domain (GGDEF) and an active PDE domain (EAL) by a HAMP signal transduction module (Figure 1B). We have recombinantly expressed and purified the GGDEF and EAL domains of BifA and have demonstrated using isothermal titration calorimetry that this construct binds GTP with a Kd of $\sim 7.7 \mu \mathrm{M}$. Using site-directed mutagenesis we have identified residues in the GGDQF sequence motif that are required for GTP binding and found that GTP binding affects the oligomeric state of BifA and its interactions with PelD. Structure determination of the cytoplasmic domains of apo-BifA and its complex with GTP are currently in progress. Conclusion: Our data suggests a model wherein the degenerate GGDEF domain of BifA functions as a GTP sensor, which in turn regulates not only BifA PDE activity, but also its interactions with target effectors such as PelD. Significance: There are thousands of DUAL GGDEF-EAL domain proteins spread across all bacterial phyla. Deciphering the interplay between the GGDEF and EAL domains of BifA will aid in the understanding of other DUAL GGDEF-EAL domain proteins that participate in c-di-GMP metabolism to regulate important physiological responses. Additionally, understanding how the PDE activity of BifA is regulated by GTP binding will be key to understanding how it interacts with PelD and exerts its specific control over Pel biosynthesis in P. aeruginosa. References: 1. Jackson, K.D. et al. (2004). J. Bacteriol., 186(14), 4466-4475. 2. Franklin, M.J. et al. (2011). Front. Microbiol., 2. 3. Abdallah, M. et al. (2014). Arch. Microbiol., 196, 453-472. 4. Mulcahy, L.R. et al. (2014). Microb. Ecol., 68(1), 1-12. 5. Jennings, L.K. et al. (2015). PNAS, 112(36), 11353-11358. 6. Friedman, L. and Kolter, R. (2005). Mol. Micro., 51(3), 675-690. 7. Lee, V.T. et al. (2007). Mol. Micro., 65(6), 1474-1484. 8. Kuchma, S.L. et al. (2007). J. Bacteriol., 189(22), 8165-8178.

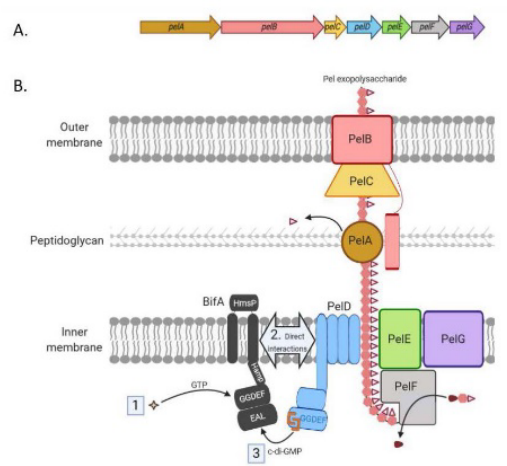

Figure 1. Model of the BifA-dependent down-regulation of the P. aeruginosa pel biosynthetic complex. A: Schematic of the pel operon. B: Schematic outlining the proteins involved in the polymerization (PelDEFG), modification (PelA) and export (PelBC) of the Pel exopolysaccharide and the role of BifA in regulating Pel biosynthesis. The biosynthesis of Pel is regulated posttranslationally by the binding of c-di-GMP to the inhibitory site of the degenerate GGDEF domain of PelD. The binding of GTP to the GGDEF domain of BifA (Step 1) is proposed to cause a conformational change such that BifA can sequester c-di-GMP from PelD through direct interactions (Steps 2 and 3), ultimately down-regulating the production of Pel.

Acta Cryst. (2020). A76, a159 